FORMATION Formation emploi

Revue française de sciences sociales

107 | juillet-septembre 2009

La formation professionnelle en Amérique latine

\title{
Un dispositif d'insertion des jeunes : contextes et acteurs dans les nouvelles stratégies d'action publique. Le cas du programme « pro-jeunes » en Uruguay
}

Integrating young people: context and players in the new public-administration strategies. Example of the youth promotion programme in uruguay

Eine Maßnahme zur Eingliederung junger Menschen : Hintergründe und Akteure in den neuen öffentlichen Handlungsstrategien. Der Fall des „ProJugend"-Programms in Uruguay

Un dispositivo de inserción de jóvenes : contextos y actores en las nuevas estrategias de acción pública. El caso Projoven en Uruguay.

\section{Claudia Jacinto}

Traducteur : Mireille Zangani

\section{OpenEdition Journals}

Édition électronique

URL : http://journals.openedition.org/formationemploi/1997

DOI : 10.4000/formationemploi. 1997

ISSN : 2107-0946

Éditeur

La Documentation française

Édition imprimée

Date de publication : 1 septembre 2009

Pagination : 41-55

ISSN : 0759-6340

Référence électronique

Claudia Jacinto, « Un dispositif d'insertion des jeunes : contextes et acteurs dans les nouvelles stratégies d'action publique.

Le cas du programme " pro-jeunes » en Uruguay », Formation emploi [En ligne], 107 | juillet-septembre 2009, mis en ligne le 01 juin 2011, consulté le 30 octobre 2020. URL : http://journals.openedition.org/ formationemploi/1997 ; DOI : https://doi.org/10.4000/formationemploi.1997 


\title{
DOSSIER
}

\section{Un dispositif d'insertion des jeunes: contextes et acteurs dans les nouvelles stratégies d'action publique. Le cas du programme «pro-jeunes » en Uruguay}

Claudia Jacinto*

\begin{abstract}
Afin de perdurer, le dispositif "pro-jeunes » s'est appuyé sur un système tripartite $d^{\prime}$ 'acteurs et une source de financement permanent; il a su assurer sa légitimité auprès des jeunes comme de certaines niches professionnelles, et a obtenu de bons résultats en termes d'égalité des opportunités.
\end{abstract}

Un des débats récurrents sur les dispositifs d'aide à l'insertion des jeunes a trait aux changements des formes d'action publique qu'ils représentent. Les alliances, les complémentarités et les tensions entre les différents acteurs ont fait l'objet d'études et de débats.

En Amérique latine, l'apparition de ces dispositifs dans les politiques actives d'emploi, depuis environ quinze ans, correspondait à des critiques sur la formation professionnelle en vigueur à ce momentlà. Celle-ci apparaissait bureaucratisée et réitérait davantage sa propre offre qu'elle ne répondait aux nouvelles exigences de compétentes sur le marché de l'emploi. Pour autant, elle devait se transformer en un modèle " piloté par la demande ». Ainsi, entrèrent
* Claudia Jacinto est docteur en sociologie, spécialiste de l'Amérique latine, de l'université Paris III, France. Coordonnatrice de redEtis, de I'IIPE (Institut international de planification éducative)-UNESCO, et chercheure au CONICET (Conseil national de la Recherche d'Argentine) à I'IDES (Institut de développement économique et social). Elle a publié récemment : Jacinto C. (2008) "Latin america's efforts in the vocational training of young people from poor backgrounds" in Atchoarena David y Grootings Peter (Eds.) UNEVOC Handbook of VET Reforms. Jacinto C. (2006) "Estrategias sistémicas y subjetivas de transición laboral de los jóvenes en Argentina. El papel de los dispositivos de formación para el empleo." Revista de Educación, n $n^{\circ} 341$, Ministerio de Educación, Cultura y Deporte de España. 
en vigueur des dispositifs d'insertion des jeunes de bas niveau de qualification ${ }^{1}$ qui constituaient un des groupes à fort taux de chômage.

Souvent, ces initiatives ont été éphémères, selon les gouvernements en place ou les sources et les orientations du financement externe (crédits multilatéraux ou coopération bi-latérale). La question majeure de cet article a trait aux contextes et raisons pour lesquelles certains dispositifs ont réussi à se pérenniser, se constituant en une stratégie d'action publique avec une relative stabilité. Pour discuter et illustrer cette question, nous examinerons soigneusement le cas d'un de ces dispositifs en Uruguay, Pro-jeunes (Pro-Joven).

Afin de contextualiser les caractéristiques de ce programme dans les systèmes de formation et de travail latino américains, il convient d'évoquer brièvement l'expérience récente dans la région dans ce domaine.

Depuis les années 50, l'offre de formation professionnelle initiale destinée aux jeunes n'accédant pas à l'école secondaire s'appuyait sur les Instituts tripartites de formation professionnelle ou sur les centres de formation professionnelle (FP) dépendant des ministères de l'Éducation, particulièrement dans les cas de l'Argentine et de l'Uruguay. Les classes sociales qui accédaient à la plupart de ces centres conventionnels étaient en général celles qui, au moins potentiellement, pouvaient s'insérer dans les secteurs formels de l'économie, en général les fils d'ouvriers industriels, puisque ce développement était lié aux processus de développement industriel de l'après-guerre. ${ }^{2}$ Dans les années 70 , ces Instituts de FP commencèrent à offrir aussi de la formation plus courte pour le secteur informel, c'est-à-dire les petits établissements qui emploient moins de cinq personnes (qui souvent ne payent pas d'impôts) ruraux et urbains. Cette formation, remarquable du point de vue de sa capacité à former pour un métier susceptible de générer des revenus, était cependant de moindre qualité que celle destinée à former les

\footnotetext{
${ }^{1}$ Les jeunes de bas niveau de qualification sont ceux qui n'ont pas atteint le niveau d'enseignement secondaire (12 ans de scolarité), soit environ $65 \%$ des jeunes latino-américains.

${ }^{2}$ Actuellement, presque la moitié de la force de travail latinoaméricaine s'insère dans des emplois informels (CEPAL, 2005).
}

ressources humaines pour le secteur formel de l'économie (Jacinto, 2008). En bref, la formation professionnelle est différenciée et segmentée : d'un côté, des circuits d'une qualité et d'une exigence élevées, liés aux secteurs productifs et aux métiers compétitifs, avec une participation des entreprises pour ceux qui possèdent un niveau secondaire complet; de l'autre, des circuits de qualités et ressources moindres, dans des locaux communs, destinés aux emplois informels à faible productivité3 .

Au cours des années 90, cette situation se complique avec l'ajout de nouveaux cours ad hoc, liés aux politiques actives d'emploi ou aux programmes sociaux de "lutte contre la pauvreté » (Jacinto, 2006). Il s'agissait de mesures de "formation et d'accompagnement » (selon la typologie proposée par Lefresne, 20074), coordonnés principalement par les ministères du Travail et/ou de l’Emploi et financés par la Banque interaméricaine de développement (BID) et dans certains cas, par la Banque mondiale. On adopta un modèle de « marché », caractérisé par la signature de contrats de cours de formation auprès de centres de formation professionnelle principalement privés et d'organisations non gouvernementales (ONG) et ce par le biais d'appels d'offres. Ils offraient une formation courte et souple, destinée à l'emploi formel, y compris des stages en entreprises. Plusieurs pays ont développé ces programmes: Argentine, Chili, Uruguay, Pérou, Panama, Colombie.

Le cas du dispositif Pro-jeunes montre un processus d'institutionnalisation, puisqu'il a réussi à perdurer depuis treize ans.

Quels sont les facteurs et processus expliquant la pérennité de ce modèle de formation au cours d'une période d'importantes mutations socio-politiques et du marché du travail ? Comment est-on parvenu à stabiliser un réseau d'acteurs institutionnels? Quel rôle ont joué les acteurs sociaux dans ce processus ?

\footnotetext{
${ }^{3}$ Il convient de différencier l'usage des termes «formation professionnelle » et « formation/qualification professionnelle ». Le premier est un terme plus englobant qui inclut différents niveaux de compétence, formation professionnelle de type "scolaire » et continue. Le terme "formation/qualification technique » renvoie en général à des enseignements courts et ponctuels.

${ }^{4}$ L'auteur classifie trois types de mesures : former et accompagner vers l'emploi les jeunes en difficulté ; donner accès à l'entreprise et explorer des nouveaux gisements d'emploi.
} 
Cet article s'appuie sur une étude qualitative ${ }^{5}$ visant à examiner les événements et processus qui ont caractérisé la consolidation du modèle du dispositif. Il s'intéresse au rôle des acteurs qui sont intervenus, les modalités de gestion, les changements dans le contexte socio-économique et politique et les régulations dans ces processus. Pour reconstruire ces processus, nous recourons à diverses techniques et sources de données. Parmi les sources secondaires, nous analysons une abondante information fournie par le programme (cahiers des charges des appels d'offres pendant 12 ans $^{6}$, documents internes et rapports annuels) ; évaluations disponibles et réglementation. Ainsi, nous examinons les statistiques nationales et celles du programme. Enfin, nous présentons des entretiens semi-dirigés réalisés auprès des membres de l'Unité de coordination (UDC) du programme, des représentants des entreprises, syndicats et fonctionnaires qui appartiennent au Comité national de l'Emploi (JUNAE) dont dépend le programme, de même que l'organisation de deux groupes focus de vingt institutions formatrices ${ }^{7}$.

L'article montrera d'abord la place de Pro-jeunes au sein de la formation professionnelle initiale en Uruguay et les particularités de son mode de gestion, pour examiner ensuite les étapes de développement, en se centrant sur les deux conditions qui le favorisèrent : la participation des acteurs tri-partite et les bons résultats d'insertion. Il conclut que la durée de ce dispositif et du réseau institutionnel qui s'est alors mis en place est liée à : a) à l'intérieur du régime d'éducation-travail du pays, le dispositif s'est imposé comme le seul à offrir une formation technique courte et une insertion professionnelle destinée aux jeunes de bas niveau de qualification b) le modèle de gestion met fortement l'accent sur la gestion pédagogique et a été réadapté à diverses occasions c) il a obtenu de bons résultats d'insertion, même durant les périodes de très fort chômage d) son

\footnotetext{
5 Je remercie Florence Finnegan pour sa collaboration dans l'analyse et la systématisation des données et Jorglina Sassera de son aide pour l'édition.

${ }^{6}$ Qui ont été systématiquement comparés pour appréhender les changements.

${ }^{7}$ Cette étude, en accord avec l'institution, est issue de la recherche sur « la formation professionnelle des jeunes de bas niveaux de qualification en Amérique latine » du IPPE-UNESCO, coordonnée par l'auteur.
}

financement et l'appui institutionnel dont il bénéficie proviennent d'un organisme tri-partite et permanent, et ne dépendent pas des fonds publics.

\section{LA PLACE DU PROGRAMME PRO-JEUNES DANS LA FORMATION PROFESSIONNELLE INITIALE EN URUGAY}

Dans cette partie, nous développerons les arguments concernant les deux premiers points que nous venons d'évoquer. C'est-à-dire que la stabilité relative du dispositif est due, entre autres, au fait qu'il est le seul dans le pays à offrir une qualification technique courte et une insertion professionnelle aux jeunes de bas niveau de qualification, et que son modèle de gestion a mis l'accent sur la qualité pédagogique et la flexibilité afin de s'adapter aux circonstances changeantes.

Mais afin de contextualiser le cas, il convient de signaler qu'en 2006, le PIB de l’Uruguay ${ }^{8}$ s'élevait à 19221 millions de dollars, composé du secteur des services (59\%), du secteur industriel (23\%) et du secteur agricole, de la pêche et des minerais (9\%). C'est un pays à revenus moyens et l'un des plus égalitaires d'Amérique latine (même si $20 \%$ des foyers sont considérés comme pauvres), et il se place au 43e rang en matière de taux de développement humain (PNUD) sur 177 pays.

Le régime d'éducation et de formation professionnelle (Verdier et Buechteman, 1998) est de type académique, principalement orienté vers les études supérieures. Le système éducatif est centralisé par l'Administration nationale de l'Éducation publique (ANEP). Après un enseignement primaire de 6 années, l'enseignement secondaire est organisé en un cycle basique commun et obligatoire de 3 ans, et un cycle supérieur également de 3 ans, non obligatoire. On peut suivre des cours dans des institutions qui dépendent de deux organismes déconcentrés de l'ANEP : le Conseil d'Éducation secondaire et le

\footnotetext{
${ }^{8} \mathrm{La}$ population totale du pays s’élève à 3305723 millions d'habitants, avec seulement $6,4 \%$ de population rurale.
} 
Conseil d'Éducation technique professionnelle (CEPT), qui est chargé de la formation des techniciens supérieurs, techniciens intermédiaires et travailleurs qualifiés (Finnegan, 2006). L'enseignement technique de niveau secondaire représente $20 \%$ des effectifs du niveau, selon des données de 2004.

Dans le contexte latino-américain, l'Uruguay présente de forts taux de scolarisation au niveau secondaire : $70 \%$ pour les hommes et $77 \%$ pour les femmes. Cependant, ils dépendent largement du secteur socio-économique, puisqu'ils atteignent seulement $50 \%$ pour les jeunes issus des $30 \%$ de foyers à faibles revenus (SITEAL, 2006). En outre, le pays présente de faibles taux de réussite: environ 40 \% des élèves abandonnent avant la fin de l'éducation secondaire.

La formation professionnelle de type scolaire est prise en charge par l'Université du travail (UTU), sous la tutelle du CETP (Conseil d'éducation technique professionnelle). Créée en 1942, jusqu'aux années 90 l'UTU est au centre de l'offre publique, à laquelle accédaient principalement les fils d'ouvriers, en raison de la faible valorisation sociale de la formation aux métiers manuels.

Cette situation s'est modifiée dans les années 90, quand les acteurs des entreprises et les syndicats ont commencé à intervenir dans les politiques de formation, à partir de la création de la JUNAE (Comité national de l'Emploi), dans le cadre de la Direction nationale de l'Emploi du ministère du Travail et de la Sécurité sociale (DINAE). La JUNAE constitue un organe tripartite (comprenant les représentants des syndicats, des entreprises et de l’État, à part égale), qui gère le Fonds de Reconversion professionnelle (FRL). Outre le financement de l'assurance chômage, le FRL finance des dispositifs comme celui de Projeunes (on reviendra sur cette question).

Dans ce cadre, Pro-jeunes fut créé en 1996, avec pour objectif d'offrir une formation professionnelle courte, articulée aux demandes du marché du travail. Il était destiné aux jeunes de condition modeste, de bas niveau de qualification, et son application a été décentralisée par le biais de centres de formation (ECAs), les fonctions de coordination et de supervision de leur mise en œuvre, à travers une UdeC, étant réservées à la JUNAE. Le dispositif ne vise pas la création d' "emplois d'insertion » (Alaluf, 2003) mais l'insertion dans des emplois formels.

On exige des ECAs (centres de formation) qu'ils insèrent effectivement $45 \%$ des formés dans les emplois formels ${ }^{9}$. Les cours représentent entre 250 et 500 heures, et comportent formation technique, orientation socio-professionnelle pratique en entreprise $(40 \mathrm{~h})$ et accompagnement dans le processus d'insertion. Entre 1996 et 2007, 673 cours de niveau semi-qualification ${ }^{10}$ se sont déroulés, concernant presque 20000 jeunes (Tableau 1). Les cours ont trait aux domaines administratif, vente et accueil, tourisme et hôtellerie et services aux particuliers.

Plus récemment, l'apparition de programmes sociaux liés à la création d'emplois publics temporaires, en lien avec le ministère du Développement social, a introduit de nouveaux acteurs, comme nous le verrons plus loin.

Ainsi, la formation professionnelle initiale, en partie intégrée au système éducatif, l’UTU, et en partie affiliée au ministère du Travail et à la JUNAE, connaît une institutionnalisation polyforme ${ }^{11}$, selon le concept suggéré par Agulhon (2006) pour le système français.

Pro-Jeunes vise à offrir une formation professionnelle courte, orientée par la demande, visant spécialement le secteur des services et qui ne concurrence pas l'offre institutionnelle permanente représentée par l'UTU. L'examen comparatif des deux offres corrobore cette affirmation.

L'UTU offre une formation professionnelle initiale par le biais de cours d'une durée minimale d'un an, spécialement destinée au secteur industriel. Au cours des années récentes, l'UTU a enregistré une augmentation des offres de formations destinées aux très hauts niveaux de qualification, correspondant au niveau II (supérieur, y compris les baccalauréats) et aux cours techniques tertiaires (de 2001 à 2005, ils ont augmenté de $74 \%$ (UTU, 2006)). Par

\footnotetext{
${ }^{9}$ En cas de non-respect de cette exigence, la subvention est réduite.

10 Premier degré d'initiation technique et professionnelle.

${ }^{11}$ C'est-à-dire dépendante de plusieurs organismes publics et par le biais de différents types de centres de formation.
} 
Tableau 1

Cours et jeunes concernés par le dispositif Pro-jeunes, 1996-2007

\begin{tabular}{|c|c|c|c|}
\hline Année & Appel d'offres & Nombre de cours & Nombre de jeunes \\
\hline 1996 & 1 & 20 & 446 \\
\hline \multirow{2}{*}{1997} & 2 & 22 & 550 \\
\hline 1998 & 3 & 30 & 731 \\
\hline $1999^{*}$ & 4 & 30 & 786 \\
\hline 2000 & 5 & 40 & 1038 \\
\hline 2001 & $6 a b$ & 36 & 786 \\
\hline \multirow{2}{*}{2002} & $7 a$ & 26 & 1533 \\
\hline 2003 & $7 b$ & 31 & 1648 \\
\hline \multirow{2}{*}{2004} & $8 a b$ & 66 & 1757 \\
\hline 2005 & $9 a$ & 26 & 2526 \\
\hline \multirow{2}{*}{2006} & $10 a b c$ & 46 & 3428 \\
\cline { 2 - 4 } & $11 a b$ & 110 & 4637 \\
\hline $2007^{* *}$ & $11 c$ & 31 & 19866 \\
\hline
\end{tabular}

Source : données fournies par Pro-jeunes.

* Les chiffres incluent les jeunes diplômés dans le projet Opportunité Jeunes.

** Données partielles.

Les lettres qui suivent les numéros des appels d'offres indiquent des appels d'offres différents portant le même numéro (par exemple, il y a eu trois appels d'offres $n^{\circ} 10: a$, b et c).

contre, Pro-jeunes s'est caractérisé, au fil du temps, par sa capacité à offrir des cours de moins d'une année, sélectionnés selon leur qualité et leur pertinence par le biais d'appels d'offres. Les ECAs doivent s'engager en matière d'insertion professionnelle, ce qui limite le nombre de formation qu'elles proposent.

Ainsi, l'ampleur de Pro-jeunes est bien moindre que celle de l'UTU sur le terrain de la formation professionnelle initiale. Il a concerné 21000 jeunes au cours des onze premières années, tandis qu'en 2005 l'UTU a accueilli 12367 jeunes jusqu'à 24 ans à ce niveau de formation.

Les populations concernées aussi sont différentes. Projeunes est destiné aux jeunes de 18 à 24 ans issus de foyers à bas revenus, n’ayant pas atteint le niveau secondaire. L'UTU, même si elle capte aussi une population ayant ce profil, a tendance à accueillir de plus en plus de jeunes plus âgés. Au cours de cette décennie, la participation des jeunes de moins de 20 ans tend à diminuer, de même que les formations n'exigeant pas de posséder le niveau secondaire inférieur.

Les institutions formatrices qui participent à l'une ou l'autre politique diffèrent également. L'UTU est implantée dans les centres de formation professionnelle et les écoles publiques, avec une couverture nationale. Les contenus s'appuient sur les lignes définies au plan national. Par contre, les cours de ProJeunes sont décentralisés grâce aux ECAs (centres de formation), qui sont principalement des ONG et des instituts privés de formation. Ces centres sont chargés d'identifier les domaines de formation qui 
correspondent aux demandes des entreprises et, en fonction de cela, d'élaborer leur proposition de formation. L'évaluation des propositions privilégie la qualité de l'offre technico-pédagogique, les profils des enseignants, l'articulation au marché du travail et le prix.

Ainsi, les deux offres divergent quant à leur action, leur niveau de centralisation, leur orientation vers des secteurs d'activité différents, leurs caractéristiques quant au public accueilli et par le caractère des institutions formatrices (voir tableau 2).

Cependant, un des premiers défis auquel fut confronté le développement de Pro-jeunes, comme modèle de formation flexible, a été l'apparition d'un savoir-faire en matière de gestion. À cet égard, le développement d'une unité de coordination (UdeC) dépendant d'une logique bureaucratique de contrôle, et qui combinait le contrôle administratif et l'accom-

Tableau 2

\section{Comparaison dispositif Pro-jeunes-UTU (Université technique d'Uruguay), aspects généraux et formation initiale}

\begin{tabular}{|c|c|c|}
\hline & UTU & Pro-Jeunes \\
\hline \multicolumn{3}{|c|}{ ASPECTS GÉNÉRAUX } \\
\hline Tutelle institutionnelle & $\begin{array}{l}\text { Ministère de l'Éducation - ANEP - } \\
\text { CFT (administration nationale } \\
\text { de l'Éducation publique) }\end{array}$ & Comité national de l'emploi (UUNAE) \\
\hline Caractéristiques générales de l'offre & Formation professionnelle régulée & $\begin{array}{l}\text { Cours de formation sur appels d'offres, } \\
\text { ajustés à la demande }\end{array}$ \\
\hline Niveau de qualification atteint & $\begin{array}{l}\text { Depuis le niveau I à III, majorité } \\
\text { de l'effectif dans les cours de niveaux II } \\
\text { et III }\end{array}$ & Niveau l \\
\hline Profil dominant parmi les étudiants & $\begin{array}{l}\text { Adultes de } 20 \text { ans, sans profil social } \\
\text { pré-défini }\end{array}$ & $\begin{array}{l}\text { Adultes de moins de } 21 \text { ans, de milieux } \\
\text { modestes }\end{array}$ \\
\hline \multicolumn{3}{|c|}{ FORMATION NIVEAU I DANS LES DEUX INSTITUTIONS } \\
\hline Durée des cours & $\begin{array}{l}\text { Majoritairement d'un an ou plus, } \\
\text { et plus de } 700 \text { heures }\end{array}$ & $\begin{array}{l}\text { Majoritairement moins de } 6 \text { mois, } \\
\text { et jusqu'à } 500 \text { heures }\end{array}$ \\
\hline Structure du cours & Formation technique & $\begin{array}{l}\text { Formation technique, orientation, } \\
\text { étape pratique, aide à l'insertion } \\
\text { professionnelle }\end{array}$ \\
\hline $\begin{array}{l}\text { Nombre moyen de jeunes accueillis } \\
\text { par an }\end{array}$ & 12000 & 2000 \\
\hline Spécialités & Prédominance du secteur industriel & Prédominance des services \\
\hline Développement de programmes & $\begin{array}{l}\text { Structures nationales sur des référentiels } \\
\text { de formation }\end{array}$ & $\begin{array}{l}\text { Contenus/compétences développés } \\
\text { cours par cours, sur la base de profils } \\
\text { d'emploi }\end{array}$ \\
\hline Institutions formatrices & $\begin{array}{l}\text { Centres de formation professionnelle } \\
\text { publics }\end{array}$ & $\begin{array}{l}\text { Organismes privés de formation } \\
\text { (Centres privés et ONG - Organisations } \\
\text { non gouvernementales) }\end{array}$ \\
\hline Enseignants & Fonctionnaires de l'État & $\begin{array}{l}\text { Embauchés par les ECAs } \\
\text { (Centres de formation) }\end{array}$ \\
\hline
\end{tabular}

Sources : réalisation de l'auteur sur la base de données fournies par Pro-Jeunes, UTU www.utu.edu.uy 
pagnement pédagogique, semble fournir une explication. Tous les acteurs interwievés, depuis les membres de la JUNAE jusqu'à ceux de la coordination et les ECAs eux-mêmes, sont d'accord sur ce point.

Depuis le début, l'unité de coordination (UdeC) a eu de multiples fonctions : élaboration des cahiers des charges des appels d'offres, évaluation des propositions, suivi administratif des cours et contrôle et orientation pédagogique. Fait important, tous les membres, depuis le coordinateur jusqu'aux membres techniques d'une équipe de quinze personnes, avaient des profils professionnels comportant une formation pédagogique.

L'analyse comparative des spécificités et des conditions pour répondre aux appels d'offres, au fil du temps, surprend par l'accent mis sur l'aspect pédagogique. En effet, outre les conditions formelles et légales, le modèle pédagogique auquel les cours doivent se conformer est précisément déterminé ; à cet effet, sont fournis des modèles pour la planification de l'offre et les critères d'évaluation (taux de réinscription scolaire, taux d'insertion au travail; profils des enseignants, etc.). Ainsi, la conception du dispositif est fortement prescriptive.

Cependant, cette conception a aussi fait montre d'une certaine souplesse dans l'ajustement aux conditions croncrètes de mise en œuvre, ce qui se reflète dans les modalités de contrôle et dans la reconnaissance de la construction de savoirs mutuels entre UdeC (unité de coordination) et ECAs (centres de formation).

Conformément à l'opinion partagée par l'équipe centrale et les ECAs eux-mêmes, la supervision inclut la consultation, le contrôle et l'accompagnement administratif et pédagogique (Aldaba, 2008). Elle comporte des visites des centres de formation et des entreprises où s'effectuent les stages et/ou les insertions professionnelles.

Ce modèle de contrôle a été facilité par plusieurs facteurs. En premier lieu, le nombre d'ECAs qui participent est limité : par exemple, en 2006, ils sont 27, la moyenne des cours par ECA étant de 3 cours. Cette limite facilite le suivi et le renforcement du dispositif, même si la répartition géographique constitue un obstacle. En second lieu, même si au fil du temps, des changements sont intervenus au sein des ECAs parti- cipantes, presque la moitié des ECAs participent au dispositif depuis 5 ans. En troisième lieu, la conception propre du dispositif, qui ne limite pas le nombre de cours à sélectionner, réduit la « compétence » entre les ECAs. En effet, les ECAs pouvaient collaborer entre eux dans les discussions pédagogiques et présentations devant l’UdeC. L'espace de compétence restait circonscrit à la relation avec les entreprises qui fournissaient des stages ou des emplois. Cela a facilité l'instauration de relations basées sur une logique collaborative plutôt que compétitive, du moins sur certains aspects.

L'unité de coordination et les centres de formation reconnaissent la souplesse et la capacité d'apprentissage au cours du temps. Ainsi, le coordinateur d'un ECA indique que: "(Pro-Jeunes) s'est construit avec les institutions, ce fut toujours un aller-retour. On a écouté les institutions, les jeunes et les entreprises ». L'UdeC partage cette vision, selon les dires des membres de l'équipe : "L'unité coordinatrice a appris des institutions ${ }^{12}$. Parmi les données recueillies au cours des entretiens, on observe que la création de savoirs institutionnels a concerné en particulier deux aspects a) Comment travailler avec des jeunes aux profils psycho-sociaux accueillis par le dispositif ? b) Comment développer des profils formateurs liés aux activités et comment se rapprocher des entreprises?

Dans la construction de cette logique collaborative, les ECAs font état du compromis réalisé par l’UdeC dans l'atteinte de l'objectif d'insertion des jeunes. Ainsi, un autre coordinateur d'ECA indique que : «Pro-jeunes a une souplesse d'action pour changer de stratégies, de méthodologies si c'est nécessaire, [...] pour accepter que l'institution prenne des décisions qu'elle pense nécessaires pour ses cours. Cette flexibilité relève de la clarté de l'objectif d'insertion, il ne s'agit pas de se soumettre à la rigidité d'un processus bureaucratique mais d'atteindre l'objectif ».

Pro-jeunes combine alors une conception comportant un haut degré de presciption sur le type de formation et le modèle pédagogique avec un contrôle qui «accompagne» les ECAs, ce qui favorise le

\footnotetext{
12 En effet, l'UdeC tient compte des commentaires et des évaluations des ECAs pour ajuster le programme lors de chaque appel d'offres.
} 
renforcement mutuel. Les documents du dispositif et divers auteurs externes rendent compte aussi de cet aspect (Lasida et Pereira, 1997 ; Chiodi, 2005). Ainsi, la consolidation d'un «modèle de formation qui fonctionne " peut être considérée comme une des dimensions du processus d'institutionnalisation du dispositif. Mais cette consolidation est indissolublement liée aux bons résultats d'insertion, même dans des contextes défavorables du marché du travail. Une approche historique du dispositif permettra d'approfondir cet aspect et le rôle des acteurs tri-partites.

\section{D'UN DISPOSITIF TRANSITOIRE À UN PROCESSUS D'INSTITUTIONNALISATION}

\section{La création de Pro-jeunes comme dispositif de formation, d'orientation et d'insertion (1996-1999)}

Comme nous l'avons mentionné, Pro-jeunes dépend d'un organisme tri-partite, la JUNAE (Comité national de l'Emploi), qui prend les décisions finales quant aux appels d'offres, à la sélection des cours, ainsi qu'à la participation d'autres acteurs dans le dispositif (Aldaba, 2008). Les acteurs de la JUNAE, malgré leurs divergences sur d'autres aspects, ont construit un consensus sur la continuité du dispositif et sa forme d'action ${ }^{13}$. Nous évoquons différents événements qui illustrent cette affirmation.

Le programme qui a précédé Pro-jeunes est le programme « Option Jeunes », en vigueur entre 1994 et 1997, avec un financement de la banque multilatérale. Ici on a testé la signature de contrats de cours de formation par le biais d'appels d'offres publics, adoptant une stratégie décentralisée et un engagement d'insertion des jeunes diplômés de la part du centre de formation. Quelles sont les conditions qui ont favorisé la création de Pro-jeunes comme prolongement d'Option Jeunes?

${ }^{13}$ Les appels d'offres comme les programmes et leurs évaluations doivent être approuvés par consensus de tous les représentants de la JUNAE.
Les politiques actives d'emploi et de formation professionnelle qui se sont développées dans le pays, depuis 1992, fournissent un cadre à la création et ensuite à l'institutionnalisation de Pro-jeunes. En 1996, une loi change radicalement le scénario de formation professionnelle. Elle mentionne que les FRL (Fonds de reconversion professionnelle) pourront être utilisés pour le développement de dispositifs de formation et d'emploi destinés aux populations pauvres à bas niveau de qualification, non bénéficiaires de l'assurance chômage (Chiodi, 2006) ${ }^{14}$. Projeunes fut l'un des programmes créés. Ainsi, la loi a accru l'offre de formation en autorisant le recours à des entreprises privées, alors qu'auparavant seuls les services de formation de l'Université technique (UTU) et du Centre de formation et de production (ECA) pouvaient conclure des contrats de formation (Firgueira et alii, 2002).

En 1997, une autre loi, destinée à la promotion et à l'insertion professionnelles des jeunes, a homologué des formes de contrats de travail à durée déterminée, d'une durée maximale d'un an, exonérés de charges patronales. Ainsi furent créées les conditions facilitant l'insertion des jeunes exigée par Pro-Jeunes ${ }^{15}$. Cette loi constitue un exemple intéressant qui montre comment, malgré les désaccords dans les autres domaines, les acteurs tri-partites se sont entendus pour soutenir le dispositif. Le projet de loi a été l'objet d'âpres discussions, dans lesquelles les acteurs des entreprises souhaitaient étendre les contrats à durée déterminée et les stages, tandis que les acteurs syndicaux y étaient opposés de même qu'à la gratuité des stages. Finalement, la loi fut approuvée, suivant en cela les préconisations des représentants des entreprises. Face à cette nouvelle réalité, l'acteur syndical présent dans la JUNAE exerça un contrôle strict pour que les offres de formation de Pro-jeunes garantissent que les stagiaires ne se substituent pas aux travailleurs. Par contre, du côté des entreprises, de nombreux témoignages attestent que leur participation aux discussions sur la sélection et l'évaluation des cours a été

\footnotetext{
${ }^{14}$ Très tôt, les dispositifs ont concerné les exclus de l'emploi, à la différence de qui s’est passé en France (Mauger, 2001).

${ }^{15}$ Cette loi a été très utilisée par les ECAs, si bien qu'au début 2000, $55 \%$ des contrats d'insertion ont été signés dans ce cadre (Lasida, 2002).
} 
Tableau 3

Taux de chômage total de la population active, et des jeunes, et pourcentage d'insertion professionnelle post-cours du dispositif Pro-jeunes 1997, 2002-2006.

\begin{tabular}{|c|c|c|c|}
\hline & $\begin{array}{c}\text { Taux de chômage total } \\
\text { population active }\end{array}$ & $\begin{array}{c}\text { Taux de chômage des } \\
\text { jeunes }\end{array}$ & $\begin{array}{c}\text { Pourcentage d'insertion } \\
\text { Pro-jeunes }\end{array}$ \\
\hline 1997 & 11,4 & 27,2 & $49\left(^{\star}\right)$ \\
\hline 2002 & 18,6 & 38 & $54\left(^{* \star}\right)$ \\
\hline 2006 & 12 & 30 & $86\left(^{\star \star \star}\right)$ \\
\hline
\end{tabular}

Source : Taux : Institut national de statistique d'Uruguay.

$(*)$ Extraits de Lasida et Pereira, 1997.

(**) Extraits de Naranjo, 2002.

$(* * *)$ Extraits de Pro-jeunes, 2007.

moins active. Comme de nombreux auteurs le confirment et comme cela ressort des entretiens, la faible participation du secteur entrepreunarial représenté dans la JUNAE est liée au fait que les entreprises représentées intéressées par l'embauche des diplômés des Pro-jeunes constituent des exceptions ${ }^{16}$. Les personnes interrogées dans les entreprises ont cependant indiqué qu'elles considéraient qu’il était nécessaire, d'un point de vue stratégique, de participer à la JUNAE.

Les évaluations ont confirmé l'efficacité du dispositif. L’année suivant sa création, une étude réalisée par Lasida et Pereira (1997), a révélé que 49 \% des jeunes avaient réussi à s'insérer professionnellement après leur passage par le dispositif. Les cours qui comportaient des stages se sont révélés plus efficaces en matière d'insertion professionnelle des diplômés.

S'instaure alors une combinaison entre bons effets relatifs et consensus des acteurs tri-partites, qui ont soutenu le dispositif au long des années, à tel point que, actuellement, on le considère comme « le dispositif emblématique », " celui ayant la plus grande envergure » parmi ceux financés par la JUNAE. Le tableau 3 montre l'évolution des taux d'insertion post-programme en comparaison avec les taux de chômage d'ensemble et des jeunes. Nous reviendrons sur cet aspect dans les parties suivantes.

\footnotetext{
${ }^{16}$ En revanche, ce sont les entreprises petites et moyennes qui ont embauché les diplômés de Pro-jeunes.
}

\section{La crise socio-économique et la participation de Pro-jeunes à d'autres initiatives (2000-2003)}

L'étape suivante, celle de la crise économique aigue que traverse le pays, permet de montrer comment le programme s'est adapté au nouveau contexte.

À partir de 1999, les contextes national et régional se modifient de façon drastique. La crise économique et la récession commencent à s'étendre dans le pays. Entre 1999 et 2003, le PIB (produit intérieur brut) enregistre un taux de croissance négatif, atteignant $15 \%$ fin 2002. La période est marquée par un accroissement très significatif du chômage : le taux de chômage a atteint presque $20 \%$ en 2002-2003. Quant au taux de chômage des jeunes de 14 à 24 ans, il est de $38 \%$.

Dans ce contexte, le ministère du Travail suspend la signature de contrats de cours de formation, jusqu'à 2001. Cette décision est surtout liée au désengagement financier du FRL (fonds de reconversion professionnelle), comme corollaire à l'introduction d'un taux flexible de recette dans la loi de 1996. Mais elle est aussi liée à la crise des relations entre les acteurs sociaux, en particulier entre les entreprises et les syndicats, dont les positions divergeaient sur les deux questions. En premier lieu, l'entreprise rejeta la proposition syndicale d'introduire dans les cours des modules obligatoires sur les droits et obligations des travailleurs. En second lieu, par rapport à un projet sur les standards et normes en matière de compétences professionnelles et leur certification, les syndicats 
soutinrent que cette fonction revenait aux travailleurs et aux employeurs ; le secteur entrepreneurial souhaitait intégrer d'autres acteurs, comme les dirigeants d'entreprises, les usagers, les consommateurs, les centres de formation, etc. En raison de ces divergences, les deux questions firent l'objet de querelles.

Malgré cette crise, la JUNAE a soutenu le maintien de Pro-jeunes grâce à diverses stratégies. D'un côté, des cours étaient approuvés par anticipation, mais il y avait aussi des Fonds provenant d'autres sources. En effet, profitant de l'existence d'une UdeC (unité de coordination) disposant d'une expérience technique et pédagogique, d'autres départements publics invitèrent Pro-jeunes à participer à la réalisation d'autres programmes de formation professionnelle. Par exemple, Opportunité Jeunes, soutenu par la banque multilatérale. Un autre exemple est la participation des Travailleurs en assurance chômage (TSD) dans la composante « Jeune » du dispositif. C'est-à-dire que les jeunes bénéficiant de l'assurance chômage suivaient une formation de Pro-jeunes en contrepartie. Afin d'adapter la proposition aux jeunes les plus vulnérables, des ateliers d'aide à la réinsertion professionnelle, de formation à l'auto-emploi et de formation en alternance furent développés. Comme nous l'avons vu dans le tableau 1, ces stratégies permirent à Pro-jeunes d'accroître le nombre de jeunes accueillis par rapport aux années précédentes.

Dans le même temps, au cours de cette période, un nouveau pas fut franchi en direction de l'institutionnalisation des ECAs. Au début du programme, afin de stimuler le développement des institutions de formation, les cours étaient payés au-dessus des prix du marché. D’un côté, cela a favorisé le développement des ECAs, mais d'un autre, certaines d'entre elles étaient de petites institutions, de qualité douteuse. En 2000, on introduit des conditions plus drastiques en matière de participation des ECAs grâce à la mise en place du Registre Unique des Centres de Formation.

Parmi d'autres facteurs (politiques, budgétaires), la continuité paraît basée d'une part sur le consensus des acteurs tri-partites concernant la pérennité du programme, et d'autre part sur la capacité opératoire et d'innovation de l'UdeC, qui put répondre au défi de participer à d'autres dispositifs.
Au cours de ces années, on commence à reconnaître à Pro-jeunes une certaine expertise dans la conception et la mise en œuvre de dispositifs d'emploi des jeunes. En effet, dans ce contexte de crise, les effets sur l'insertion professionnelle des diplômés se révèlent comparativement bons, selon deux évaluations de la période (Naranjo, 2002 ; SIPRON, 2003), la probabilité de trouver un emploi à la fin de la formation étant de 54 \% (tableau 3). D'une certaine façon, les jeunes formés et ceux du groupe de contrôle avaient des taux de chômage identiques. Malgré cela, en matière de qualité des emplois, les différences étaient notoires : la stabilité et la formalité des emplois comme les rémunérations étaient meilleures parmi les participants que dans le groupe témoin.

\section{Changements dans les politiques actives d'emploi et dans les « dispositions » des jeunes (2004-2007)}

Dés 2003, se met en place un scénario différent pour les politiques de l'emploi. La tendance à l'augmentation du chômage s'inverse.

Cependant, pour les jeunes, les difficultés d'emploi persistent, comme illustré par le tableau 3. En outre, certaines caractéristiques du marché du travail constituent un obstacle aux possibilités d'intervention des dispositifs de type Pro-jeunes, même en période de reprise économique. D’un côté, davantage que les adultes, les jeunes occupent un emploi précaire (seulement un peu plus de la moitié des jeunes occupés bénéficiaient en 2006 de la sécurité sociale). D'un autre côté, la discrimination ethnique et/ou sociale est souvent associée aux jeunes rencontrant des problèmes de chômage plus importants. Pour autant, l'objectif du dispositif, «formation, orientation et aide à l'insertion », peine à contrer les caractéristiques structurelles du marché du travail. Dans le même temps, la demande de travailleurs plus qualifiés est croissante, réduisant les opportunités des moins qualifiés. Ceux qui ont un niveau secondaire incomplet représentent $65 \%$ des sans-emploi. Face à cette situation, les politiques éducatives encouragent le maintien dans la scolarité secondaire, et incitent au retour à l'école. 
Avec l'arrivée de la gauche à la présidence en 2005, des changements de perspective se produisent aussi dans les politiques sociales, d'autres programmes « entrant en concurrence " avec Pro-jeunes. Un ministère du Développement social est créé, et dans ce cadre se mettent en place un programme « Revenu minimum d'insertion citoyen » destiné aux populations les plus pauvres et un programme de travail transitoire appelé «Travail pour l’Uruguay ». Dans ce dernier, une composante appelée "Travail pour Uruguay Jeunes » accueillait les jeunes inscrits âgés de 18 à 24 ans. Les jeunes devaient réaliser des activités sociales dans une ONG pendant 5 mois, 4 heures par jour, en contrepartie du RMI. En outre, ils devaient participer à 60 heures mensuelles de formation. Ces programmes s'inscrivaient dans les dispositifs attribuant un revenu de substitution en contrepartie de l'acceptation d'un emploi temporaire dans des activités d'intérêt général (Alaluf, 2003). Comme ce qui s'est passé pour des dispositifs identiques en Europe, les emplois étaient liés aux « emplois de proximité », c'est-à-dire aux besoins sociaux dans le secteur non commercial.

L'introduction de ce type de programme a eu une influence sur Pro-Jeunes puisque les territoires et les populations cibles se superposent dans une certaine mesure. Pour les jeunes, les nouveaux programmes représentent l'opportunité d'accéder rapidement à un revenu et aussi à une formation, même de moindre valeur que celle prévue par Pro-joven. Les témoignages recueillis chez les ECAs indiquent que pour le jeune ces choix impliquent «moins d'efforts » et changent la relation coût/bénéfice de la participation à Pro-jeunes. Ainsi, pendant ces années, le nombre de jeunes qui se présentaient aux convocations a diminué. Pour les institutions formatrices, il devenait plus aisé d'obtenir une formation sans l'engagement d'insertion professionnelle exigé par Pro-jeunes. Elles purent ainsi décider de participer à l'un ou l'autre des programmes, ou à plusieurs à la fois.

Parallèlement, les conséquences socio-culturelles de deux décennies sans amélioration des conditions de vie et de crise socio-économique, conjuguées à l'intensification des problématiques sociales et l'éclosion des cultures jeunes ont influé également sur Pro-jeunes. Tous les acteurs interrogés évoquent des changements dans les profils psycho-sociaux des jeunes accueillis et de leur relation au travail. Selon ces acteurs, ce changement de comportement se reflète dans ce qu'ils appellent « la faible motivation pour le travail». Se produit alors un déplacement dans la compréhension du problème : des besoins de formation vers les "dispositions des jeunes sans emploi ", comme le propose Mauger (2001), le risque étant la responsabilisation individuelle du problème (Tanguy, 2008).

La reprise économique augmente les ressources du FRL (fonds de reconversion professionnelle) et renforce le rôle des acteurs sociaux syndicaux et des entreprises face à l'État (dont la faiblesse est qu'il ne dispose pratiquement pas d'autres fonds pour les politiques actives d'emploi). Un autre élément renforce le lien entre ces deux acteurs sociaux face à l'État : prétendant renforcer le rôle de l'État, la représentation étatique propose que les décisions cessent d'être prises au consensus mais le soient à la majorité. Les syndicats et les entreprises ne sont pas d'accord. Ce désaccord a constitué, entre autres, un obstacle à la création de l'Institut National de l'Emploi et de la Formation.

Cependant, les programmes continuent à se dérouler. Les acteurs tri-partites conviennent d'insister plus fortement pour que l'insertion des jeunes s'opère sur le marché formel du travail, en n'acceptant pas la formation et/ou l'insertion sur des emplois de très basse qualification, comme les services domestiques. Même si la négociation peut être âpre, en général les cours proposés par l’UdeC sont approuvés. La représentation syndicale continue à avoir le rôle le plus actif dans la sélection des propositions. Elle exige que les entreprises candidates ne soient pas en conflits du travail ou légaux avec les travailleurs et veille à la qualité des insertions. Le rôle des entreprises devient moins actif dans la définition des conditions des cours. Plusieurs interviewés indiquent qu'on doit faire en sorte que les secteurs entreprenariaux représentés dans la JUNAE n'exigent pas une main-d'œuvre de niveau semi-qualifié, comme les diplômés de Pro-jeunes. Cependant, les entreprises prétendent qu'elles continuent à soutenir Pro-jeunes en raison de l'engagement d'insertion qui s'avère différent de celui de l'UTU et pour les bons résultats d'insertion. 
Quels sont les changements qui sont intervenus dans la conception de Pro-jeunes face à ces nouveaux contextes ? Dès 2003, l'accompagnement s'accentue grâce à une stratégie personnalisée au cours du processus d'insertion professionnelle. La pertinence attribuée aux insertions éducatives s'accroît. En 2005, on tend davantage vers la réussite de l'intégration sociale et citoyenne des jeunes, à partir de nouveaux éléments de la proposition formative qui consistent à «souligner les compétences transversales (...) compétences clés pour l'employabilité et l'exercice de la citoyenneté qui incluent des compétences basiques (lecture-écriture et calcul), interpersonnelles (comme communication, relationnel, travail en équipe et autres), technologies, informatique et toute autre compétence qui contribue à l'employabilité » (selon les cahiers des charges des appels d'offres).

Comme on le voit, le modèle a su se montrer flexible, non seulement comme le résultat d'apprentissages dans la relation UdeC-ECAs, mais aussi par les changements dans la configuration de politiques sociales et éducatives, dans les cadres légaux, dans les équilibres entre les acteurs de la JUNAE, dans les profils des jeunes accueillis, etc.

Quels sont les résultats d'insertion dans cette nouvelle étape ? L'enquête 2006 de suivi des diplômés (ESE) révèle des résultats incontestables en matière d'insertion des diplômés. 86 \% ont trouvé un emploi sur le marché formel, tandis que seulement $53 \%$ des jeunes travailleurs uruguayen âgés de 18 à 24 ans occupent des emplois formels. De même, le dispositif a eu un effet positif sur les jeunes qui au début du dispositif n'étudiaient pas et ne travaillaient pas ; ils sont passés de $64 \%$ au moment de l'inscription au programme à 26 \% au moment de l'enquête. Les «dispositions » à l'égard de l'emploi ont-elles changé ? De la même façon, 18 \% s'étaient réinscrits dans l'éducation formelle, ce qui montre aussi un impact du dispositif sur l'augmentation des opportunités éducatives. Ces données constituent les meilleurs résultats d'insertion dans l'histoire du dispositif, correspondant aussi à une phase de forte baisse du chômage dans le pays. Un des paradoxes intéressants de Pro-jeunes est que, s'agissant d'un dispositif destiné aux jeunes de faible qualification, ou compensatoire, il a eu un effet sur l'équité des opportunités professionnelles. En effet, un jeune dont le profil correspond au programme a de meilleures chances d'obtenir un emploi formel que s'il ne passe pas par celui-ci. Cela se vérifie aussi par rapport au rôle des institutions formatrices dans l'obtention des emplois ultérieurs des jeunes: autour de $40 \%$ des premiers emplois des jeunes ont été obtenus par leur intermédiaire (ESE, 2007) ${ }^{17}$. Cependant, on peut supposer que, pour les profils d'entrée des jeunes et le caractère de la formation, il s'agit d'une amélioration de l'employabilité à court terme.

ous avons tenté, au fil de cet article, de répondre à l'interrogation sur les raisons et les conditions pour lesquelles un dispositif d'insertion professionnelle des jeunes réussit à se transformer en une action publique pérenne, fondée sur un réseau de nouveaux acteurs institutionnels dans le champ de la formation.

Dans le cas examiné, nous avons vu que les réponses sont liées au fait que le dispositif a) a pu compter sur un appui politique des acteurs tri-partites et une source de financement permanente b) son Unité de coordination (UDC) a réussi, au long des années, à asseoir une double expertise vis-à-vis des attentes des jeunes eux-mêmes, de même qu'une adéquation aux exigences de certaines niches professionnelles c) a pu s'adapter à des conditions et contextes sociopolitiques et économiques différents et d) a obtenu de bons résultats relatifs en termes d'égalité des opportunités. Et même sa faible ampleur a représenté une force.

Peut-on considérer le dispositif comme un type d'action publique fixé dans la trame de la formation initiale des jeunes dans le pays?

Plusieurs éléments doivent être considérés pour apporter une réponse à cette question. L'institutionnalisation d'un dispositif comme Pro-jeunes a impliqué le renforcement d'une trame institutionnelle encore à l'œuvre. Les facteurs qui ont contribué

\footnotetext{
${ }^{17}$ En principe, un jeune peut participer une seule fois à Pro-jeunes. Mais les autres dispositifs n'ont pas connaissance de sa participation. Ainsi, on ne peut affirmer qu'on est face à une logique « d'apprentissages perpétuels » (Mauger, 2001).
} 
à le soutenir pourraient aussi constituer sa faiblesse au moment d'entrer dans un système articulé de formation et d'emploi.

Pro-jeunes a promu un modèle de formation «piloté par la demande », qui conduit les ECAs (centres de formation) à se rapprocher des entreprises employeuses potentielles. Ce procédé s'est transformé en un "savoir-faire » dans la construction de profils professionnels. Mais dans le même temps, il a conduit à la spécialisation des ECAs dans le secteur des services, et à ce qu'ils rencontrent des difficultés dans l'offre de formation destinées aux nouvelles niches professionnelles, ou dans certaines régions du pays. La recherche d'opportunités d'insertion « une à une » fonctionne à une certaine échelle mais ne suffit pas à répondre aux demandes sectorielles ou territoriales naissantes. Le modèle Pro-jeunes se confronte alors aux limites que lui impose la faiblesse des systèmes d'information et d'intermédiation, principalement au niveau local et régional.

Comme on l'a vu, Pro-jeunes occupe une place au sein du système de formation pour l'emploi, se différenciant tant de l'offre de l'UTU (Université technique) que des autres dispositifs sociaux liés à l'emploi et à l'insertion sociale. Ses bons résultats en matière d'insertion professionnelle constituent sa force. Mais le financement des formations par les appels d'offres rend fragile l'assise financière des institutions. Serait-il possible d'envisager un mécanisme d'institutionnalisation flexible qui permette le soutien des institutions et l'accumulation des apprentissages, de même qu'une souplesse pour se rapprocher de niches professionnelles de niveau semiqualifié d'insertion des jeunes?

La situation et la perspective d'évolution du marché du travail, de même que les changements dans les trajectoires des jeunes appellent à de meilleures articulations institutionnelles entre l'éducation formelle, basique, secondaire, l'UTU, construisant des « ponts » et un accompagnement à cette insertion. La mise en place de modèles de formation séquentielle, articulés avec d'autres acteurs participant à l'offre de formation pour le travail, ${ }^{18}$ en particulier l'UTU, paraît nécessaire pour proposer un objectif d'apprentissage cumulatif. Cependant, jusqu’à présent, Pro-jeunes et l'université technique ont opéré en parallèle, même quand leurs complémentarités étaient évidentes.

Ainsi le dispositif, qui était à l'origine un dispositif transitoire, s'est constitué en une forme d'action publique d'une efficacité avérée en matière de formation professionnelle des jeunes de bas niveau de qualification, mais sa valorisation et sa pérennité dépendent de la reconnaissance de son rôle au sein de la reformulation des politiques de formation et d'emploi. La création d'un Institut national de l'Emploi tri-partite et articulant formation et emploi est mis à l'agenda politique du pays, et on envisage la construction d'un système d'éducation, de formation et de certification de compétences professionnelles. Dans ce cadre, la nouvelle place de Pro-jeunes sera évoquée.

Pouvons-nous tirer les enseignements de cette expérience quant à l'institutionnalisation d'un dispositif de ce type dans d'autres contextes? La réponse s'avère ambiguë. D'un côté, l'analyse que nous venons de réaliser fournit quelques clés sur ce qui a conduit à ce processus. Elle montre qu'un dispositif comme celui-ci peut contribuer à l'équité et peut façonner un modèle d'action publique durable. D'un autre côté, cette même analyse montre que les aspects structurels et socio-culturels propres au pays, la construction et la dynamique de son régime d'éducation et de travail, de même que sa propre culture politique, ont conduit à cette évolution.

Traduit de l'espagnol au français par Mireille Zangani (Céreq), avec l'aimable collaboration de l'auteur.

\footnotetext{
${ }^{18}$ C'est-à-dire qu'un jeune peut suivre un cours de niveau I dans le dispositif Pro-jeunes et suivre une formation de niveau II à l’université technique.
} 
Aldaba J. A. (2008), Con la mira en lo publico: una aproximación desde las redes de política al programa projoven, tesis de licenciatura, universidad de la República, Montevideo.

Agulhon C. (2006), "Les relations formationemploi: un serpent de mer ", in Revue Questions Vives, état de la recherche en éducation, vol. $3, \mathrm{n}^{\circ} 6$, pp. 39-50, université de Provence, Lambesc.

Alaluf M. (2003), Evoluciones del desempleo, políticas del empleo e inserción de los jóvenes, universidad Libre, Bruselas.

Cepal (2005), Panorama social de América Latina 2005, Naciones Unidas, Santiago de Chile.

Chiodi F. (2005), Políticas de empleo y formación profesional en Uruguay 1992-2005, OIT; MTSS ; Italia Laboro, Cooperazione Italiana, Montevideo.

ESE (2007), Presentación de resultados de la Encuesta 2006 de seguimiento de egresados, Montevideo, Projoven.

Filgueira F., Errandonea F., Porzecanski Y R. (2002), "Negociación colectiva y políticas de formación profesional en el Uruguay. Capital, trabajo y Estado en el nuevo modelo de desarrollo", en A. C. Posthuma (coord.) Diálogo social, formación profesional e institucionalidad, Cinterfor/OIT, Montevideo. Disponible en www.ilo.org/public/spanish/ region/ampro/cinterfor/publ/posthu/pdf/1_3.pdf

Finnegan F. (2006), Reseña de políticas educativas públicas de articulación de la educación media con la formación para el trabajo en América Latina. Los casos de Colombia, Uruguay, México, Brasil y Chile, redEtis-IIPE-UNESCO, Buenos Aires.

Jacinto C. (2006), "Los caminos de América Latina en la formación vocacional de jóvenes en situación de pobreza. Balance y nuevas estrategias”, en Claudia Jacinto, Cristina Girardo, María de Ibarrola y Prudencio Mochi, Estrategias educativas y formativas para la inserción social y productiva, pp. 87105, Cinterfor/OIT, Montevideo.

Jacinto C. (2008), “La transición laboral de los jóvenes y las políticas públicas de educación secundaria y formación profesional en América Latina: ¿qué puentes para mejorar las oportunidades?” En Espinosa B., Estéves A. y Pronko M., Mundos del trabajo y políticas públicas en América Latina, FLACSO, Quito.

Lasida J., Pereira Y J. (1997), “Projoven: encuentro y negociación entre la capacitación y el mercado de Uruguay" en Boletín Técnico Interamericano de Formación Profesional, n 139-140, pp. 183-217, Cinterfor/OIT, Montevideo.

Lasida J. (2002), Evaluación de la aplicación de Ley de empleo Juvenil, Instituto Nacional de la Juventud (mimeo), Junta Nacional de Empleo, Instituto Nacional de la Juventud.

Lefresne F. (2007), «Les jeunes et l'emploi : parcours et dispositifs » in Les jeunes : questions de société, questions de politique, pp. 45- 75, París, «Études », La Documentation française.

Mauger G. (2001), « Les politiques d'insertion. Une contribution paradoxale à la déstabilisation du marché du travail ", Actes de la recherche en sciences sociales, 136-137, pp. 5-14, CAIRN, Paris.

Mercier D., Supervielle Y.-M. (2006), "Los métodos de difusión y apropiación de las herramientas de gestión en el proceso de globalización. Dos estudios en los extremos de América Latina: México y Uruguay” en Sociología del Trabajo, nueva época, $n^{\circ}$ 58. pp. 63-81, Siglo XXI de España Editores.

Naranjo S. (2002), Capacitación y formación profesional para jóvenes en Uruguay: los programas Opción Joven y Projoven a través de sus experiencias de evaluación, Cinterfor/OIT, PNUD, Montevideo. 
Notaro J. (2006), "Las políticas de empleo en los países del MERCOSUR, 1990 - 2003. La perspectiva regional”, en Revista de Trabajo, nueva época, año 1, n ${ }^{\circ}$ 1, pp. 61-76, Ministerio de Trabajo, Empleo y Seguridad Social, Buenos Aires.

PROJOVEN (2007), Encuesta de seguimiento egresados Projoven, disponible en http://www.projoven. gub.uy

SIPRON (2003), Evaluación de Impacto del Programa Projoven y Programa Piloto Trabajadores en Seguro de Desempleo Componente Joven, Montevideo.

SITEAL (2006), Informe sobre tendencias sociales y educativas en América Latina, OEI, IIPE-UNESCO, SITEAL, Buenos Aires.

Strauss A. y Corbin J. (2002), Bases de la investigación cualitativa. Técnicas y procedimientos para desarrollar la teoría fundamentada. Bogotá: CONTUSEditorial Universidad de Antioquia (2a. ed.).
Tanguy L. (2008), « La recherche de liens entre la formation et l'emploi : une institution et sa revue. Un point de vue », Formation Emploi, n ${ }^{0} 101$, pp. 23-39, Cereq, La Documentation française, Paris.

Universidad del Trabajo del Uruguay- Utu (2006), Análisis cuantitativo de la matrícula de UTU, año 2005. Informe especial panorama de la matrícula 1996-2005, UTU, Montevideo.

Verdier E. y Buechteman C. (1998), "Regímenes de educación y de formación profesional: evidencia macroinstitucional” en Gautié J. y Neffa J. (comps.) Desempleo y políticas de empleo en Europa y Estados Unidos, Trabajo y Sociedad - CEILPIETTE/ CONICET - Lumen/Humanitas, Buenos Aires.

Verdier E. (2008), « L'éducation et la formation tout au long de la vie : une orientation européenne, des régimes d'action publique et des modèles nationaux en évolution ", Sociologie et Sociétés, vol. 40, n 1 , université de Montréal, Montréal.

Résumé

$$
\begin{gathered}
\text { Un dispositif d'insertion des jeunes : contextes et acteurs } \\
\text { dans les nouvelles stratégies d'action publique. } \\
\text { Le cas du dispositif « pro-jeunes » en Uruguay } \\
\text { Claudia Jacinto }
\end{gathered}
$$

Cet article s'intéresse aux dispositifs d'insertion des jeunes en Amérique latine, qui sont parvenus à se transformer en une stratégie stable d'action publique mobilisant de nouveaux acteurs du champ de la formation. On examine le cas du programme Pro-jeunes en Uruguay. On montre que sa pérennité pendant plus d'une décennie est liée au fait que le dispositif a) s'est construit comme le seul à offrir de la formation professionnelle courte, notamment aux jeunes de bas niveaux de qualification, b) son modèle de gestion met fortement l'accent sur l'aspect pédagogique et a été réaménagé à différentes occasions, c) il a obtenu de bons résultats d'insertion même dans les périodes de très fort chômage d), son financement émane d'un organisme tri-partite, permanent, et ne dépend pas des Fonds publics. Cependant, l'évolution du marché du travail et les changements dans les trajectoires des jeunes exigent de meilleures articulations institutionnelles entre l'éducation formelle, la formation professionnelle et l'orientation socioprofessionnelle dans le cadre d'un système intégré.

Mots clés

Jeune, insertion professionnelle, mesure jeune, bnq - bas niveau de qualification, Uruguay

Journal of Economic Literature: J 24 